Gut, 1980, 21, 753-759

\title{
Gastric motility and emptying in normal and post-vagotomy subjects
}

\author{
H J SHEINER,* M F QUINLAN, AND I J THOMPSON \\ From the Department of Surgery, University of Western Australia and Department of Nuclear Medicine, \\ Sir Charles Gairdner Hospital, Perth, Western Australia
}

SUMMARY The effects of proximal gastric vagotomy (PGV) and vagotomy with pyloroplasty ( $V$ and $P$ ) on gastric motility were studied using a solid meal labelled with a radiopharmaceutical agent. In having on-line computer facilities it was possible not only to record the rate of emptying but also to analyse the relative roles of the fundus and the antrum within the overall framework of gastric emptying. In normal subjects the fundus filled and then emptied in an almost linear pattern. The antrum, however, did not completely fill until well after the meal was eaten and thereafter appeared to maintain a constant volume during the study. The redistribution of contents between fundus and antrum was reflected in the total stomach emptying curve as a delay, or lag phase before gastric emptying commenced. After both types of vagotomy fundic filling was delayed, representing a slower eating time, which was presumably due to early satiety. Antral filling and volume was disturbed only after $\mathrm{V}$ and $\mathrm{P}$, which was also reflected by a loss of the lag phase seen on the total stomach curve. PGV retained antral function but there was significant delay in the redistribution of contents between fundus and antrum, though this did not have clinical significance. The rate of emptying was unaffected by either operation. It was concluded PGV did maintain antral function and a more normal pattern of emptying compared with $\mathbf{V}$ and $\mathrm{P}$. After $\mathrm{V}$ and $\mathbf{P}$ the changes in antral function were considerable and these changes are probably associated with some of the complications resulting from this operation.

The reduced incidence of post-vagotomy dumping and diarrhoea is the distinct advantage of proximal gastric vagotomy (PGV) over vagotomy with a drainage procedure $(\mathrm{V}$ and $\mathrm{P}) .^{1-3}$ It has been assumed that this improvement is attributable to the preservation of antroduodenal motility with maintenance of normal control over gastric emptying. The evidence for this has come mainly from animal experiments, ${ }^{45}$ whereas in human subjects the results are conflicting. Proximal gastric vagotomy has been shown to increase the rate of emptying of fluid meals, ${ }^{67}$ whereas with a nutritional contrast medium meal there is a normal rate of emptying. ${ }^{8}$ Other workers, using food labelled with a radiopharmaceutical agent, showed temporary delay in the rate of emptying but the rate rapidly returned to normal within a month of surgery. ${ }^{910}$ Recently, a persistently

-Address for correspondence and for reprints: Associate Professor H J Sheiner, University Department of Surgery, Queen Elizabeth II Medical Centre, Nedlands, 6009 Western Australia.

Received for publication 11 March 1980 slow rate of emptying of solid food after PGV has been shown. ${ }^{11}$

One reason for the discrepancy in results lies in the methods which are available to assess gastric motility. ${ }^{12}$ Non-invasive techniques are highly desirable in the clinical situation but these usually assess motility by simply measuring the rate of emptying as expressed by the half emptying time $\left(\mathrm{T} \frac{1}{2}\right)$. There have been few attempts to assess the overall pattern of gastric emptying and, in particular, to look at the early phase of emptying, which may be crucial to the understanding of postvagotomy complications. ${ }^{1314}$ Also, few studies have been undertaken in which the relative roles of antrum and fundus are assessed within the overall framework of gastric emptying.

The present study was undertaken in order to clarify the changes in gastric motility which follow either a PGV or V and $P$ in man. A non-invasive technique was used and the rate of gastric emptying determined by conventional means. In addition, the pattern of emptying was studied by analysing 


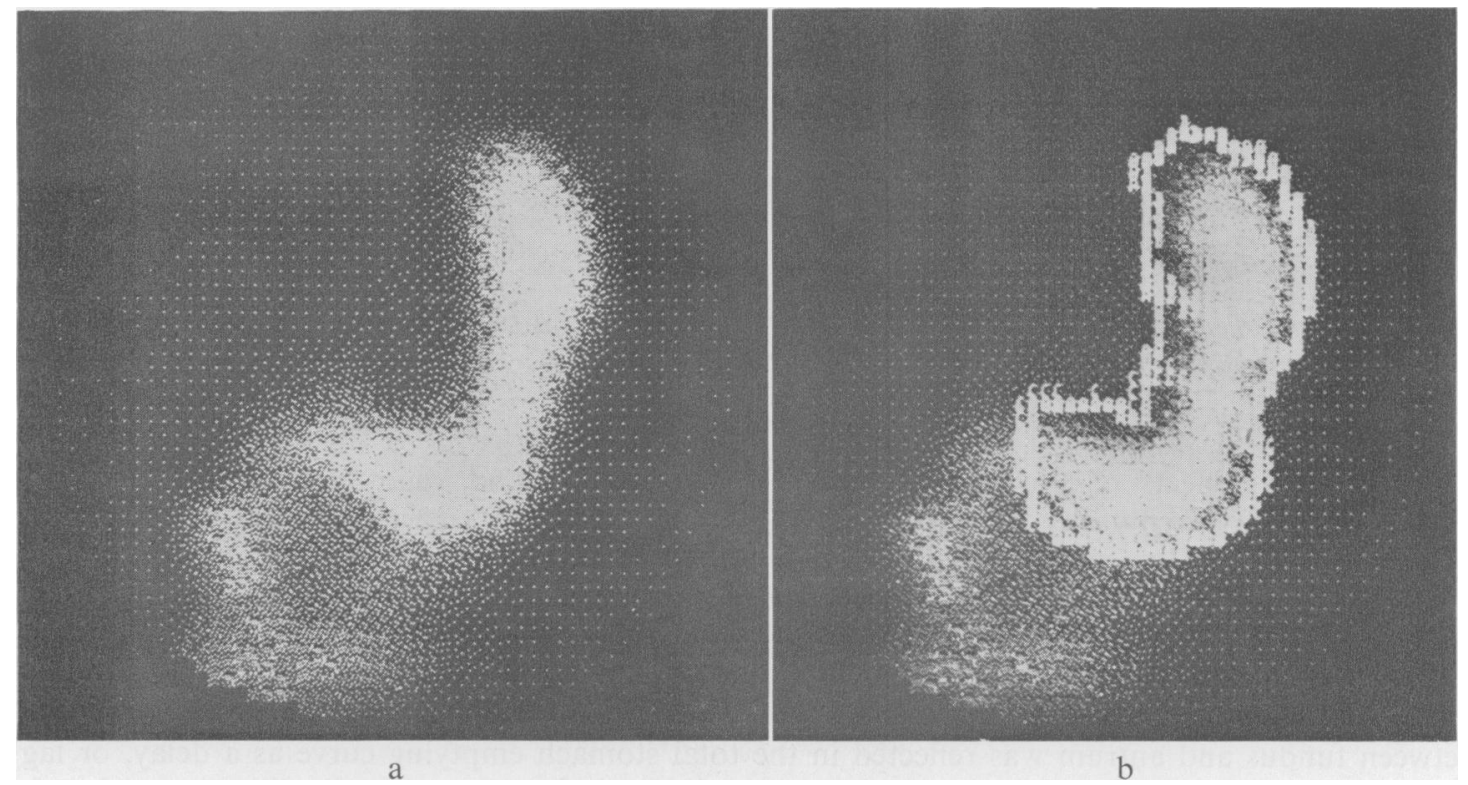

Fig. 1 The gastric image obtained by adding a combination of the 120 frames collected during the study (a). The total gastric area was then outlined as well as proximal and distal subareas representing antrum and fundus (b). Thus, three areas of interest were obtained.

the changes which occurred within the fundus and antrum of the stomach.

\section{Methods}

The method of gastric emptying has been described previously. ${ }^{15}$ A meal consisting of a standard volume and weight of a minced meal and mashed potato meal labelled with $1 \mathrm{mCi}$ of $99 \mathrm{~m} \mathrm{Tc}$ macroaggregates of ferrous hydroxide was given to the fasting patient. The meal is regarded as 'solid', because, although it has a 'cottage pie' consistency, the stomach has less mechanical mixing to do than it does with a meal of large slices of solid food. The isotope was firmly bound to the food and was not absorbed or adsorbed onto the mucosa. ${ }^{15}$ The meal was given with the patient seated upright with the collimator of a Toshiba Gamma Camera placed against the upper abdomen. Counts were collected at 30 second intervals for one hour from the time of beginning the meal, and all data were stored on disk via an on-line PDP 11/40 computer.

\section{ANALYSIS OF DATA}

At the end of the study an image of the stomach was outlined by playing back any one or combination of frames (Fig. 1a). From this, areas of interest were outlined for subsequent analysis. The regions outlined were the total stomach area and the proximal and distal halves (Fig. 1b). These latter two areas may not be completely anatomically accurate but a consistent narrow waist between the two was seen in most studies and it is believed that they represent activity within the fundic and antral portions of the stomach.

Having selected the areas of interest it was then possible to obtain time/activity curves reflecting the activity within these three areas during the time of study (Fig. 2).

To assess the rate of emptying, two cursor points were placed on the total stomach curve, the first being at the time emptying was seen to start and the second when the study ended at 60 minutes. Between these two points an exponential curve was fitted from which the $T \frac{1}{2}$ was automatically calculated (Fig. 3). The computer was programmed to allow for instrument dead time and isotope decay.

The normal gastric emptying pattern showed several constant features with distinct differences in the pattern between the three curves (Fig. 2). The top curve, which represents the whole stomach, filled rapidly followed by a plateau period (lag) in which there was no evidence of emptying for some minutes. After this time there was an exponential or linear pattern of emptying. The middle curve, representing fundal activity, filled and emptied in a nearly linear pattern. The lower curve, representing the antrum, did not begin to fill for some 


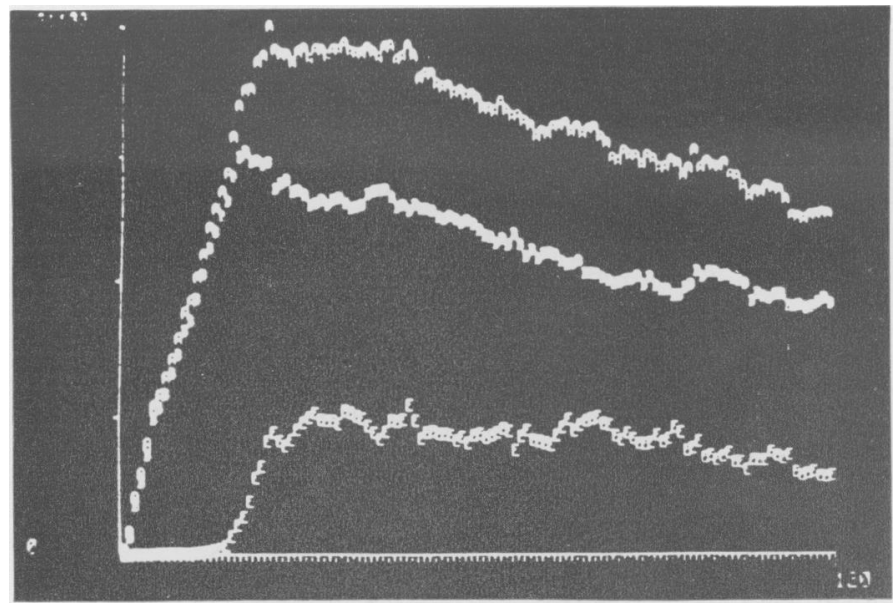

Fig. 2 Time/activity curves from the three areas of interest. The top curve represents the total stomach activity, the middle curve the fundic area and the lower curve represents antral activity. Note the different patterns between the three. The total stomach fills, then 'plateaus' before emptying; the fundus fills and then empties immediately; and the antrum fills last but then the activity remains constant suggesting controlled filling and emptying (see text).

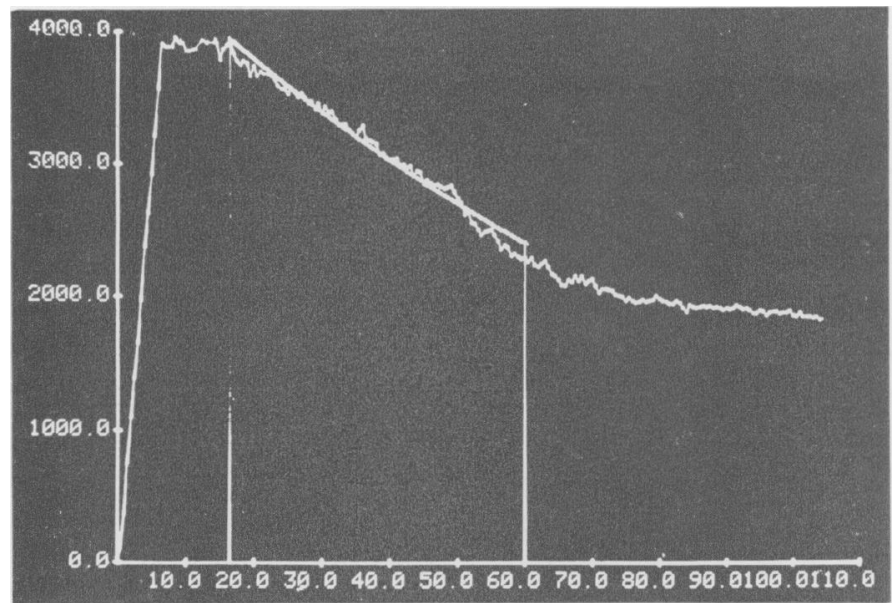

Fig. 3 Example of calculation of half emptying time $\left(T \frac{1}{2}\right)$. The first cursor point was placed where activity begins to decrease - that is, onset of emptying at 16.5 minutes-and the second cursor point at 60 minutes (end of study). In this demonstration the $T_{\frac{1}{2}}$ was 61.6 minutes with an error $E$ of 0.025 .

time and when it had filled the activity remained at a near constant level from then until the completion of the study.

To further assess these features each patient study was analysed. This analysis required observer interpretation and therefore, was called a 'subjective analysis'. The features looked for were (1) the presence of a lag phase in the total stomach curve; (2) the presence of an antral plateau; (3) evidence of a delay before the antrum began to fill.

Figure 2 also shows that it was possible to measure the duration of the above features and such measurements were called 'objective analysis'. They included (1) time to fill the fundus; (2) time to fill the antrum; (3) length of the lag phase in the total stomach curve (when present).

Eighteen normal subjects (male volunteers between the ages of 20 and 40 years were studied, with repeat studies being carried out on 11 people to assess reproducibility. Eight patients with duodenal ulceration (DU) were studied before surgery; 16 patients were studied at least six months after a vagotomy and pyloroplasty and 10 patients were studied at least three months after a proximal gastric vagotomy.

\section{Results}

\section{REPRODUCIBILITY}

The reproducibility of the $T \frac{1}{2}$ has been described elsewhere. ${ }^{15}$ It showed a significant difference between normal people ( $\mathrm{F}=4.95 ; \mathrm{P}=<0.01)$ but no difference with repeat meals in the same subject $(F<1$; $\mathbf{P}>0.05$ ). Similar results in the reproducibility of the pattern of emptying were obtained using both the subjective and objective criteria described above. 
Table 1 Visual inspection of emptying curves: 'subjective analysis'

\begin{tabular}{lccrcc}
\hline Subjects & (No.) & Lag phase & $\begin{array}{c}\text { Antral } \\
\text { plateau }\end{array}$ & $\begin{array}{l}\text { Delay before } \\
\text { antrum fills }\end{array}$ \\
\hline Normal & 18 & + & 14 & 14 & $11+$ \\
DU & 8 & - & 4 & 4 & 7 \\
HSV & 10 & - & 0 & 6 & 7 \\
V and P & 16 & - & 2 & 8 & 1 \\
& & -6 & $* 5$ & 10 \\
& & -10 & 11 & 15 \\
\hline
\end{tabular}

This shows the number of patients with $(+)$ and without $(-)$ the 'subjective' features looked for on analysis of individual curves. Note the delay in the onset of antral filling after both types of vagotomy compared with normal $(+)(P<0.03)$.

After $V$ and $P$ there were significant differences, Also, fewer $V$ and $P$ patients had an antral plateau compared with normal $(P<0.008)$, DU $(P<0.05)$, and PGV $(P<0.02)$ subjects. Fewer patients exhibited a lag phase compared with normal $(P<0.02$, DU $(P<0.004)$, and PGV $(P<0.04)$ subjects.

NB: statistical analysis by Fisher's exact method.

Table 2 Visual inspection of emptying curves: 'objective analysis'

\begin{tabular}{lclcr}
\hline Subjects & (No.) & Fundus & Antrum & \multicolumn{1}{c}{ Lag phase } \\
\hline Normal & 18 & $5.5 \pm 0.72$ & $16.8 \pm 1.0$ & $8.7 \pm 1.4$ \\
DU & 8 & $7.31 \pm 0.67$ & $15.6 \pm 1.02$ & $12.9 \pm 1.6$ \\
HSV & 10 & $9.3 \pm 1.08$ & $23.5 \pm 1.97$ & $13.9 \pm 3.7$ \\
V and P & 16 & $9.16 \pm 1.03$ & $15.9 \pm 2.1$ & $5.8 \pm 1.1$ \\
\hline
\end{tabular}

This shows the mean times ( \pm standard error of mean) in minutes for complete fundic and antral filling and also the mean length of the lag phase seen on the total stomach curve. Note that after PGV antral filling was significantly delayed compared with normal $(P<0.01)$, DU $(P<0.01)$, and $V$ and $P(P<0.01)$.

Also, fundic filling was slower after $P G V$ and $V$ and $P$ compared with normal $(P<0.01 \quad P<0.01)$ but not compared with $D U$ subjects (see Fig. 4).

Statistical analysis by Student's $t$ test.

\section{RATE OF EMPTYING}

There was no significant difference in the mean $T_{\frac{1}{2}}^{\frac{1}{2}}$ between all four groups. Results were expressed in minutes \pm 1 standard deviation. Normal $=73 \pm 7$; DU $58 \pm 6 ;$ PGV $55 \pm 8 ; \mathrm{V}$ and $\mathrm{P} 79 \pm 12$.

\section{PATTERN OF EMPTYING}

'Subjective analysis' (Table 1)

A lag phase in the total stomach curve was nearly always present in normal, DU, and PGV subjects. However, after $\mathrm{V}$ and $\mathbf{P}$ it was seen in only six out of 16 patients. Similarly, a plateau in the antral curve was maintained in only five out of 16 patients after $\mathbf{V}$ and $\mathbf{P}$, whereas it was almost invariably present in the other three groups. In five of the 11 $V$ and $P$ patients without evidence of an antral plateau, the antral counts barely rose above $1000 / 30$ seconds for the period of study compared with $20000 / 30$ seconds or more for normal subjects.
In assessing the presence of a delay in antral filling, this was present in only 11 out of 19 normal subjects, yet was seen in all patients after either type of vagotomy.

\section{'Objective data' (Table 2)}

The duration of the 'lag' phase in the whole stomach curve was similar in the normal and $V$ and $P$ groups but was considerably longer after PGV and in DU subjects. Note, however, that the mean time shown for the lag phase in $V$ and $P$ patients is the mean from only those five out of the 16 patients in whom

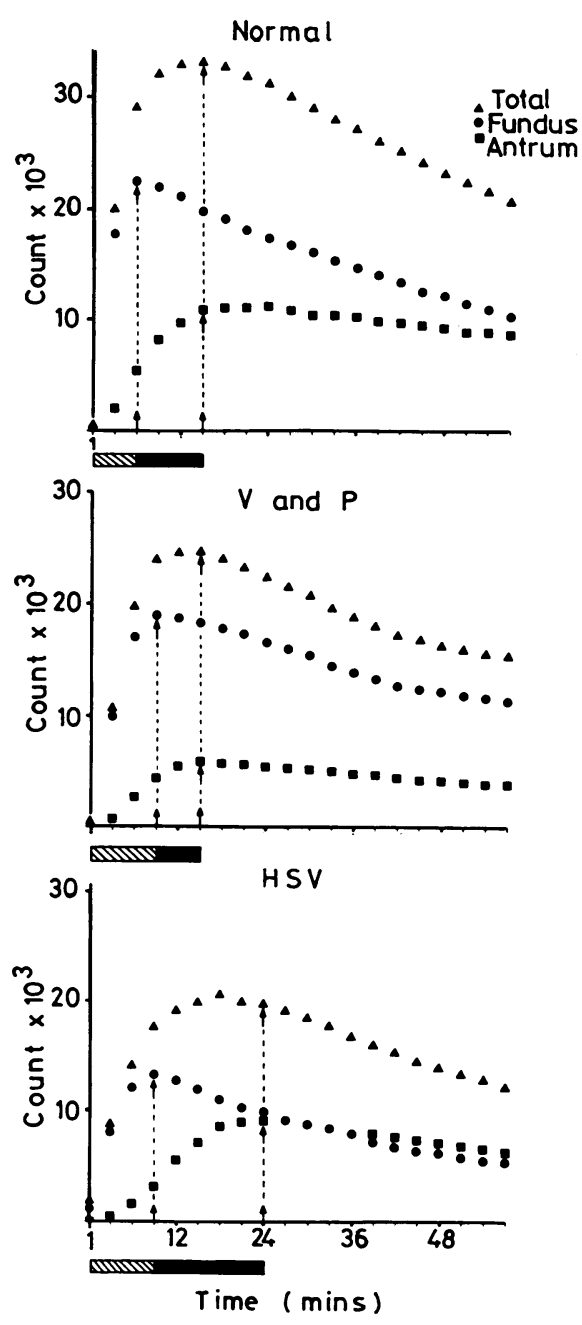

Fig. 4 Computer print-out of mean curves from normal, $V$ and $P$, and $P G V$ groups. Note the longer fundic filling after $V$ and $P$ and $P G V$ compared with normal. Also note the longer period for complete antral filling after PGV (see Table 3). 
it was present. If the other 11 patients without a lag phase were included, the mean lag phase for the $\mathrm{V}$ and $\mathbf{P}$ group was significantly shorter. However, it was decided that this might obscure the fact that there appeared to be two subgroups-a large number without evidence of a lag and a smaller subgroup with a normal pattern. The possible reasons for two such subgroups after $\mathbf{V}$ and $\mathbf{P}$ has not been examined further in this study.

The time for complete filling of the fundus was significantly slower in the postoperative patients than in the other two groups. In measuring fundic filling it was noted that, in all cases, this peak coincided with the time taken to eat the meal.

Antral filling was considerably delayed only after PGV and not in the other three groups of patients. However, it should be noted that after $V$ and $P$ only a minority of patients showed discrete antral filling and the normal antral plateau and filling times relate only to this selected minority. The reasons for this are similar to those in the analysis of the lag phase.

The differences in filling of the fundus and antrum are seen in Fig. 4 which is a computer print-out of the mean curves between all three groups of patients. This demonstrates the longer fundic filling time after vagotomy and the significantly longer antral filling after PGV. It can also be seen from Fig. 4 that there appeared to be consistently lower counts in the antral area after vagotomy and pyloroplasty.

\section{Discussion}

The method used for this study had the advantage with other isotope techniques of being non-invasive and involved minimal discomfort for the patient. However, two additional advantages of this method emerge. Firstly, data collection began immediately the patient started eating so that information was obtained as soon as food reached the stomach. Therefore, no assumptions were needed to interpret the early phase of gastric emptying. Secondly, the resolution and use of an on-line computer allowed the collection of a larger number of data points for analysis from areas within the stomach. This permitted a study of the pattern of emptying within the stomach, although the methods used to interpret this pattern may require refinement. In particular, the question of 'subjective' analysis relies on observer error. However, there was good reproducibility with replicate meals and original observations have been made in normal and postoperative people.

The normal pattern of emptying appears to be as follows. After ingestion, food immediately enters the fundus, which then fills, and continues to fill until the meal is complete. From then on the fundus empties in a linear pattern. During the fundic filling phase food leaves this area and begins to fill the antrum. The latter then fills to a certain level before emptying into the duodenum. Thus, complete antral filling does not occur until some time after finishing the meal so that there is a delay before food leaves the stomach. In other words, the 'lag' phase seen on the whole stomach curve is a reflection of the redistribution of food from the fundus to the antrum. Once gastric emptying starts, it also seems to be linear because of the unique ability of the antrum to maintain a constant volume-similar amounts enter from the fundus as leave the antrum.

In the normal person the fundic filling time coincided with the time taken to eat the meal, but it is of interest that the total stomach 'filling' continues for some minutes after the patient has stopped eating. This was interpreted as being due to a different depth relationship of portions of the stomach. As the more posteriorly placed fundus emptied into the more anteriorly placed antrum the radioactive contents came closer to the detector which, according to the inverse square law, resulted in greater efficiency of radiation detection. This explains the apparent increase in counts after the total quantity of isotope was ingested.

The rate of emptying was unaffected by either type of vagotomy, a result in keeping with other published reports..$^{10}$ In a recent study, gastric emptying was found to be delayed six to eight months after PGV.11 These authors measured gastric emptying by determining the time taken for the amount of meal remaining in the stomach to be reduced to 75,50 , and $25 \%$ respectively. As their study also suggested that a larger proportion of the meal remained in the proximal stomach, presumably they were measuring a delay in the onset of emptying but not necessarily a slower rate of emptying once the contents began to leave the stomach. Their findings are similar to the present study where a significant delay was found in the redistribution of contents between fundus and antrum after PGV, but with a subsequent normal rate of emptying.

The other effects of vagotomy on the pattern of emptying can also be explained. The longer fundic filling time relates to a longer eating time which, in turn, is presumably due to the loss of receptive relaxation after vagotomy ${ }^{16} 7$ and early satiety. The loss of the 'lag' phase in most patients after $V$ and $P$ coincides with the large number of patients in this group who are incapable of adequately filling the gastric antrum. This loss of antral 'volume' 

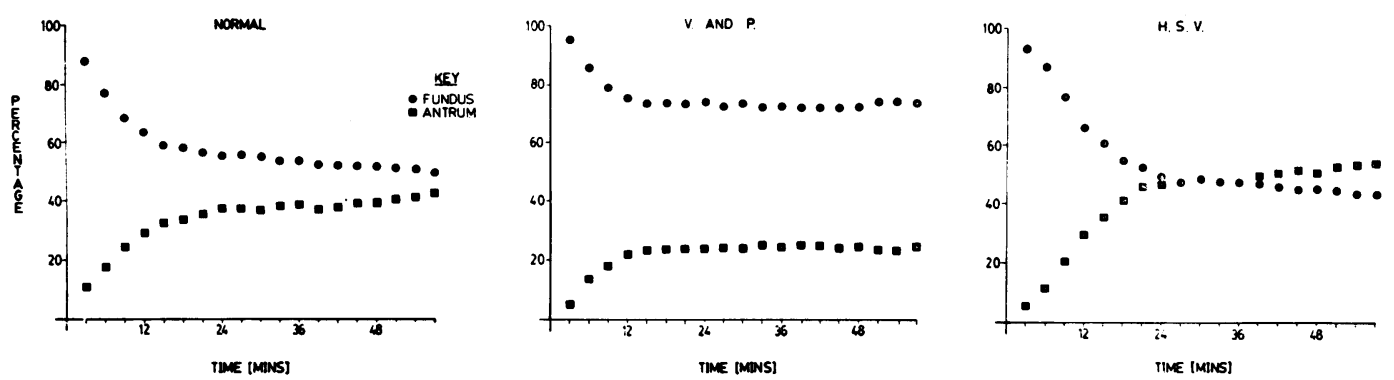

Fig. 5 The activity in fundus and antrum expressed as a percentage of total counts. Note that, in the normal PGV groups, the proportions within each compartment approached each other and remained approximately equal. After $V$ and $P$ the proportion in the antrum never exceeded $25 \%$ of the total.

is shown in a different way in Fig. 5 where the amount of radioactivity in the fundus and the antrum is expressed as a percentage of the total activity. Note that patients after PGV preserve antral 'volume' as well as showing a 'lag' phase on the total curve.

This study was not designed to correlate the abnormal patterns of emptying with post-vagotomy symptoms of dumping and diarrhoea. While there was a suggestion that the worst symptoms occurred in those patients without evidence of antral filling, the numbers were not large enough to reach statistical significance. However, previous work does suggest that the early phase of gastric emptying may be important in the determination of the cause of post-vagotomy symptoms of dumping and diarrhoea, and the present method may prove to be an acceptable one to confirm this theory.

\section{ROLE OF VAGUS IN GASTRIC EMPTYING}

From these results it is possible to interpret the role of the vagus in the normal pattern of filling and emptying of the stomach. In the filling phase the volume of the meal can be readily accepted by the fundus within six minutes, because of its receptive relaxation. This ability is lost after both types of vagotomy, leading to epigastric fullness even with the small volume of meal used in these studies, so that a mean of nine minutes is taken to eat the food and fill the fundus. Although in normal subjects some food enters the antrum, in the first few minutes there is no pooling of the meal in the most dependent part of the stomach but, rather, a controlled delivery from the fundus over the next six to nine minutes. This fundic-antral coordination is delayed after vagotomy of the fundic region (with both vagotomy and pyloroplasty and proximal gastric vagotomy).

When the antrum is vagally denervated and the pylorus destroyed (after vagotomy and pyloroplasty), the antrum is unable to retain the food.
Total emptying then begins almost as soon as the fundus empties into the antrum. On the other hand, after proximal gastric vagotomy, the controlled emptying of antral contents is maintained, although antral filling has been considerably delayed. Thus, antral innervation subserves the maintenance of an adequately filled antrum and the regular delivery of food into the duodenum. Proximal gastric vagotomy does disturb the redistribution of contents between fundus and antrum. As the antrum is of prime importance in the control of gastric emptying of solid food, the maintenance and preservation of a functional antrum is desirable. Compared with vagotomy and pyloroplasty, proximal gastric vagotomy appears to do this very well despite some disturbance in the redistribution of contents between the fundus and the antrum.

\section{References}

${ }^{1}$ Madsen P, Kronberg O. A double blind trial of highly selective vagotomy without drainage and selective vagotomy with pyloroplasty in the treatment of duodenal ulcer (abstract). Scand J Gastroenterol 1973; 8: suppl 20, 12-3.

${ }^{2}$ Johnston D. Highly selective vagotomy. Gut $1974 ; 15$ : 748-57.

${ }^{3}$ Makey DA, Tovey FI, Heald RJ. Results of proximal gastric vagotomy over 1-5 years in a district general hospital. Br J Surg 1979; 66: 39-42.

${ }^{4}$ Griffith CA, Harkins HN. Partial gastric vagotomy: an experimental study. Gastroenterology 1957; 32: 96-102.

${ }^{5}$ Amdrup E, Griffith CA. Selective vagotomy of the parietal cell mass. Part I: with preservation of the innervated antrum and pylorus. Ann Surg 1969; 170: 207-14.

${ }^{6}$ Møberg S, Carlberger G, Bàràny F, Lundh G. Gastric emptying in peptic ulcer patients before and after partial gastrectomy and selective proximal vagotomy. Rendic Gastroenterol 1972; 4: 1-7.

${ }^{7}$ Wilbur BG, Kelly KA. Effect of proximal gastric, complete gastric and truncal vagotomy on canine 
gastric electrical activity, motility, and emptying. Ann Surg 1973; 178: 295-303.

${ }^{8}$ Wilkinson AR, Johnston D. Effect of truncal, selective and highly selective vagotomy on gastric emptying and intestinal transit of a food-barium meal in man. Ann Surg 1973; 178: 190-93.

${ }^{\circ}$ Cowley DJ, Vernon P, Jones T, Glass HI, Cox AG. Gastric emptying of solid meals after truncal vagotomy and pyloroplasty in human subjects. Gut 1972; 13: 176-81.

${ }^{10}$ Howlett PJ, Sheiner HJ, Barber DC, Ward AS, PerezAvila CA, Duthie HL. Gastric emptying in control subjects and patients with duodenal ulcer before and after vagotomy. Gut 1976; 17: 542-50.

${ }^{11}$ Faxén A, Alpsten M, Cederblad A, Kewenter J, Rossander $\mathbf{L}$. The effect of parietal cell vagotomy and selective vagotomy with pyloroplasty on gastric emptying of a solid meal. Scand J Gastroenterol 1978; 13: 727-33.

${ }^{12}$ Sheiner HJ. Gastric emptying tests in man. Gut 1975; 16: $235-47$.

${ }^{13}$ Colmer MR, Owen GM, Shields R. Pattern of gastric emptying after vagotomy and pyloroplasty. $\mathrm{Br}$ Med $J$ 1973; 2: 448-50.

${ }^{14}$ Clarke RJ, Alexander-Williams J. The effect of preserving antral innervation and of a pyloroplasty on gastric emptying after vagotomy in man. Gut 1973; 14: $300-7$.

${ }^{15}$ Hamilton SG, Sheiner HJ, Quinlan MF. Continuous monitoring of the effect of pentagastrin on gastric emptying of solid food in man. Gut 1976; 17: 273-9.

${ }^{16}$ Køster N, Madsen P. The intragastric pressure before and immediately after truncal vagotomy. Scand $J$ Gastroenterol 1970; 5: 381-3. 